\title{
Improved SAGE II cloud/aerosol categorization and observations of the Asian tropopause aerosol layer: 1989-2005
}

\author{
L. W. Thomason ${ }^{1}$ and J.-P. Vernier ${ }^{2}$ \\ ${ }^{1}$ NASA Langley Research Center, Hampton, Virginia, USA \\ ${ }^{2}$ Science Systems and Applications, Inc., Hampton, Virginia, USA \\ Correspondence to: L. W. Thomason (1.w.thomason@nasa.gov) \\ Received: 10 July 2012 - Published in Atmos. Chem. Phys. Discuss.: 19 October 2012 \\ Revised: 27 March 2013 - Accepted: 2 April 2013 - Published: 3 May 2013
}

\begin{abstract}
We describe the challenges associated with the interpretation of extinction coefficient measurements by the Stratospheric Aerosol and Gas Experiment (SAGE II) in the presence of clouds. In particular, we have found that tropospheric aerosol analyses are highly dependent on a robust method for identifying when clouds affect the measured extinction coefficient. Herein, we describe an improved cloud identification method that appears to capture cloud/aerosol events more effectively than early methods. In addition, we summarize additional challenges to observing the Asian Tropopause Aerosol Layer (ATAL) using SAGE II observations. Using this new approach, we perform analyses of the upper troposphere, focusing on periods in which the UTLS (upper troposphere/lower stratosphere) is relatively free of volcanic material (1989-1990 and after 1996). Of particular interest is the Asian monsoon anticyclone where CALIPSO (Cloud-Aerosol Lidar Pathfinder Satellite Observations) has observed an aerosol enhancement. This enhancement, called the ATAL, has a similar morphology to observed enhancements in long-lived trace gas species like CO. Since the CALIPSO record begins in 2006, the question of how long this aerosol feature has been present requires a new look at the long-lived SAGE II data sets despite significant hurdles to its use in the subtropical upper troposphere. We find that there is no evidence of ATAL in the SAGE II data prior to 1998. After 1998, it is clear that aerosol in the upper troposphere in the ATAL region is substantially enhanced relative to the period before that time. In addition, the data generally supports the presence of the ATAL beginning in 1999 and continuing through the end of the mission, though some years (e.g., 2003) are complicated by the presence of episodic enhancements most likely of volcanic origin.
\end{abstract}

\section{Introduction}

The Stratospheric Aerosol and Gas Experiment (SAGE II) provided ozone, $\mathrm{NO}_{2}$, water vapor concentration and multiwavelength aerosol extinction coefficient profiles from the mid-troposphere through the stratosphere during a mission that lasted from October 1984 through August 2005. These measurements have provided crucial data in assessing the state of ozone and aerosol trends (e.g., SPARC, 2006) and remain a valuable resource to understand changes in the stratosphere and upper troposphere (UTLS). The Cloud-Aerosol Lidar Pathfinder Satellite Observation (CALIPSO) has made global $532 \mathrm{~nm}$ aerosol backscatter profiles since June 2006. While primarily focused on aerosol and thin clouds in the troposphere, its measurements have nonetheless provided a wealth of information on polar stratospheric clouds (e.g., Pitts et al., 2009) and aerosol in the stratosphere and upper troposphere (e.g., Vernier et al., 2011a). Studies of the upper troposphere and stratosphere using the combination of SAGE II and CALIPSO have been performed (Vernier et al., 2011b) which have demonstrated both the challenges and the potential for these studies. While both make measurements of attributes of aerosol, the parameters are not completely interchangeable (multiwavelength aerosol extinction coefficient versus aerosol backscatter coefficient and depolarization). In addition, sampling rates are vastly different and, perhaps the most critical complicating factor, there is no overlap period between the two instruments. Overall, it has been shown (using GOMOS as an intermediary) that there is broad consistency between the measurements of SAGE II and CALIPSO though a direct comparison is not possible. 
In Vernier et al. (2011b), the presence of an enhancement in aerosol backscatter within the Asian monsoon anticyclone (the Asian Tropopause Aerosol Layer; ATAL) was identified for the first time using CALIPSO observations. While highly variable in intensity from year to year, CALIPSO observations establish that it appears as early as May and persists as late as September but is primarily a June/July/August (JJA) phenomenon and is strongly associated with the Asian monsoon anticyclone. The spatial extent was between 14 and $18 \mathrm{~km}$, between roughly 15 and $35^{\circ} \mathrm{N}$ and between about 0 and $150^{\circ} \mathrm{E}$. While CALIPSO observations show the presence of ATAL, there is no evidence of enhanced depolarization suggesting that the increase in backscatter is likely to be in the form of liquid aerosol rather than solid aerosol. However, beyond this inference, there is little in the CALIPSO measurements themselves to suggest the source or composition of the aerosol. Some balloon-borne measurements from Tibet may support the presence of the ATAL in the same time frame prior to the CALIPSO observation; however they may also reflect volcanic activity (Tobo et al., 2007; Kim et al., 2003). On the other hand, up to this time, no analysis of SAGE II data has suggested the presence of the ATAL. This is not totally surprising due to a number of hurdles in this analysis. Observations by SAGE II in the subtropics occur at a relatively low rate even by occultation standards, and, after mid-2000, SAGE II captured only one occultation per orbit (rather than two) due to an issue with azimuth prepositioning (ordering the instrument to face the direction where an event would occur). In addition, the identification of events influenced by the presence of clouds is particularly important in the tropics and subtropics. At the same time, identifying cloud events at any latitude/altitude is subtle; missing even very small cloud effects can have an overwhelming effect on aerosol analyses in the troposphere. A previous effort to separate between cloud and purely aerosol observations by SAGE II has been shown to miscategorize some cloud events as aerosol (SPARC, 2006), and any effort to detect the ATAL in the SAGE II data must rectify that error.

Herein, we summarize challenges to separating between pure aerosol and mixed cloud/aerosol observations using SAGE II observations. In light of these challenges, we describe a new cloud/aerosol identification method developed to more effectively discriminate between cloud/aerosol events than early methods (Kent et al., 1993, 1998). Using this improved tool, we perform analyses of the upper troposphere, focusing on periods in which the UTLS is relatively free of volcanic material (1989-1990 and after 1996), and particularly attempt to detect the presence of the ATAL. Even in these nominally clean periods, small volcanic events occasionally complicate the interpretation of the analyses. As will be demonstrated below, we find that the data generally does not support the existence of the ATAL prior to 1999 but indicate its presence in following years up to the end of the SAGE II mission in 2005.

\section{Characteristics of SAGE II aerosol extinction coefficient measurements in the UTLS}

While robust and reliable in the stratosphere, the interpretation of SAGE II aerosol extinction coefficient measurements in the upper troposphere/lower stratosphere (UTLS) are complicated by the presence of clouds and a sample size that decreases within increasing depth into the troposphere. In order to evaluate aerosol properties in the UTLS, measurements influenced by cloud presence must be identified and excluded from the analysis. If this process is done effectively, some regions, particularly those associated with the western Pacific warm pool, have few observations below $14 \mathrm{~km}$. We observe that the transition between SAGE II cloud and aerosol measurements is a continuum, rather than manifesting itself as discrete modes, primarily due to the geometry of the measurements. As a result, our ability to distinguish between these two types of observations is not clear cut. Ultimately, cloud/aerosol discrimination relies on an empirical assessment of the expected behavior of mixed field of views of clouds and aerosol. The process we have developed is discussed below.

\subsection{Multiwavelength extinction coefficient reliability}

SAGE II aerosol extinction coefficient measurements have been demonstrated to be robust in the stratosphere even at the low levels observed the early 2000s with measurement uncertainties at $1020 \mathrm{~nm}$ that are often less than $10 \%$ (Thomason et al., 2008). The credit for the quality of these measurements lies substantially with the solar occultation method employed by the SAGE series of instruments. The long paths and bright radiant target (the Sun) allow a small field of view (FOV) and high vertical resolution while still producing a robust extinction signature. For example, in the clean period after 2000 , between the tropopause and $28 \mathrm{~km}, \sim 50 \%$ of the signal at $1020 \mathrm{~nm}$ is due to aerosols (with rest due to molecular scattering) and $1020 \mathrm{~nm}$ extinction coefficient values, which are between $10^{-5}$ (near $28 \mathrm{~km}$ ) and $3 \times 10^{-4} \mathrm{~km}^{-1}$ (near $15 \mathrm{~km}$ ), remain reliable to extinction at least as low as $5 \times 10^{-6} \mathrm{~km}^{-1}$ (Thomason et al., 2008). The shorter wavelength aerosol channels are not as robust due to a combination of increased molecular scattering (following wavelength to the fourth power) and absorption by gases such as ozone. The shortest wavelength aerosol extinction coefficient measurements at 386 and $452 \mathrm{~nm}$ do not reliably extend below 16 and $12 \mathrm{~km}$, respectively, and they are not particularly useful to this study. On the other hand, the measurements made at $525 \mathrm{~nm}$ are reliable in the UTLS and available as low as $5 \mathrm{~km}$ despite substantial impacts by ozone absorption and molecular scattering. The inclusion of this channel is crucial since it contributes significantly to the information content of SAGE II aerosol measurements specifically allowing a rough measure of aerosol "size". 


\subsection{Spatial sampling}

For an instrument in a mid-inclination, low-Earth orbit such as SAGE II, spacecraft sunrise and sunset occultation events occur $\sim 30$ times per day along two latitude circles that slowly progress between roughly $70^{\circ} \mathrm{S}$ and $70^{\circ} \mathrm{N}$ over the course of 4 to 6 weeks. Following a gap in SAGE II operations covering the last third of 2000 , only one event type was acquired per day and sampling was reduced to $\sim 15$ events/day for the remainder of the SAGE II mission. Due to the sampling provided by the solar occultation technique and its concomitant month-long period to complete a sweep of latitude, spatial/temporal resolution of aerosol extinction coefficient depictions (or other components of the SAGE II measurement ensemble) is limited. The sampling becomes even more restricted below the tropopause (particularly in the tropics) as many SAGE II events (profile sets) are terminated by the presence of clouds. Event termination, often (imprecisely) called "saturation", can also come about due to thick aerosol (e.g., the post-Pinatubo period of mid-1991 through 1993) and molecular effects at low altitudes for the short wavelength channels, but neither of these conditions is relevant for the 525 and $1020 \mathrm{~nm}$ aerosol extinction coefficient channels in the altitude range and time periods we consider in this paper.

SAGE II can make useful measurements up to a line-ofsight (LOS) optical depth of $\sim 7$. Given the long horizontal paths the LOS rays follow through the atmosphere, at $1020 \mathrm{~nm}$, this is roughly the equivalent of a vertical aerosol optical depth for SAGE II of $\sim 0.15$. In practice, the termination altitude of a profile is not a well-defined event, so we have adopted a convention by which a profile set is terminated at the first altitude at which $1020 \mathrm{~nm}$ aerosol extinction exceeds $2 \times 10^{-2} \mathrm{~km}^{-1}$ or the LOS optical depth exceeds 7 . We find that extinction value is an effective cutoff for profiles and also isolates the data analysis from some occasional anomalous behavior (likely an instrument artifact) at altitudes below where this extinction coefficient value is initially observed (top downward). In the time period of interest, the cutoff value occurs as a distinct step function from much lower extinctions, strongly suggesting a cloud top.

Wang et al. (2003) showed that, between 1985 and 2002, the SAGE II $1020 \mathrm{~nm}$ extinction coefficient profile termination altitude is a strong function of latitude. The zonally averaged altitude at which $50 \%$ profiles are terminated runs from about $5 \mathrm{~km}$ in high to subtropical latitudes but increases rapidly in the tropics to $\sim 11 \mathrm{~km}$. The event termination altitude in the tropics shows a strong longitudinal dependence with the highest altitudes found in the deep convection zones over South America, Africa, and particularly Indonesia. As a result, sampling at all latitudes and longitudes becomes relatively sparse at lower altitudes but is particularly acute in the tropical convection areas where meaningful tropospheric aerosol analysis is not always possible. Much of the analysis shown later in this paper is based on seasonal averages spanning 1999-2005, and data availability as a function of latitude and altitude is mostly driven by the requirement that $525 \mathrm{~nm}$ aerosol extinction be available. Globally, in each season there are approximately 2500 profile sets, and essentially all of these profiles are available above $17 \mathrm{~km}$. At $10 \mathrm{~km}$ about $60 \%$ of the profiles still provide usable measurements; at $6 \mathrm{~km}$ the fraction available has dropped to about $30 \%$. The fractions are not strongly dependent on year except in the few years following the Mt. Pinatubo eruption.

\subsection{Measurement volume}

Solar occultation is well suited to making aerosol coefficient measurements in the stratosphere, where homogeneity along the LOS is not a major concern except for relatively rare events like polar stratospheric clouds. The SAGE series of instruments implement solar occultation using scans across the Sun perpendicular to the Earth's surface during each time the Sun is obscured (or occluded) by the atmosphere relative to the spacecraft as shown in Fig. 1a. The solar scans extend across the edges of the Sun, and knowledge of the location of the Sun's edges along with knowledge of the spacecraft location and time permit very high accuracy in determining the altitude of the tangent point (closest approach to the Earth's surface) along a LOS between the instrument and the Sun for each measurement. Since the Sun is observed above the atmosphere during each event, the instrument is effectively calibrated during each event and is insensitive to changes in the instrument sensitivity (count levels) throughout the $21 \mathrm{yr}$ lifetime of the instrument. The scanning pattern is shown in Fig. 1b, and it allows multiple samples $(\sim 15)$ in the $0.5 \mathrm{~km}$ altitude sampling bins. Averaging these samples reduces measurement noise and produces the LOS measurement uncertainty that is the primary source of random noise in the final data products. A feature of this scanning process is that the volume of atmosphere represented in each altitude bin is large compared to the nominal LOS volume whose rough dimensions in the tangent altitude bin are $\sim 125 \mathrm{~km}$ (along the LOS) by the FOV of $5 \mathrm{~km}$ (horizontal) by $0.5 \mathrm{~km}$ (vertical). Given a spacecraft velocity of $\sim 7 \mathrm{~km} \mathrm{~s}^{-1}$ and a concomitant movement of the tangent location, the samples at a given altitude are relatively sparsely spaced over several hundred kilometers in the horizontal direction. Based on the high quality and low measurement noise observed in the SAGE II ozone and aerosol measurements in the stratosphere, the measurement volume does not appear to impact the quality of those measurements. In the upper troposphere, where over the scale of hundreds of kilometers variations in aerosol levels and particularly cloud presence can be substantial, the resulting mean measurement could easily represent a mixture of individual variable aerosol and cloud measurements. The sampling volume of SAGE II measurements is discussed in detail in Thomason et al. (2003). 


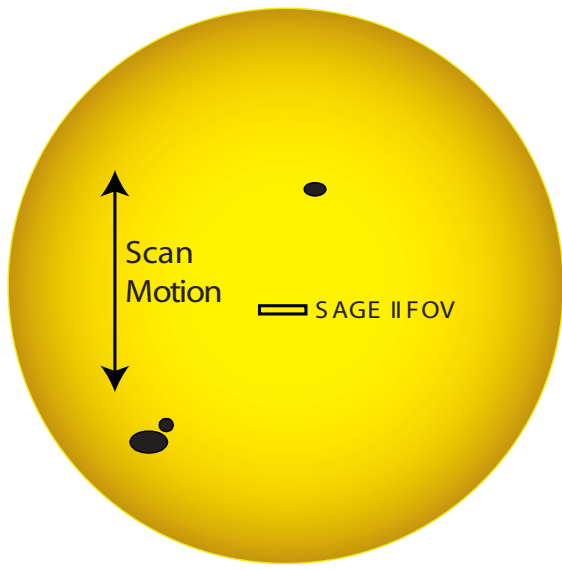

Earth

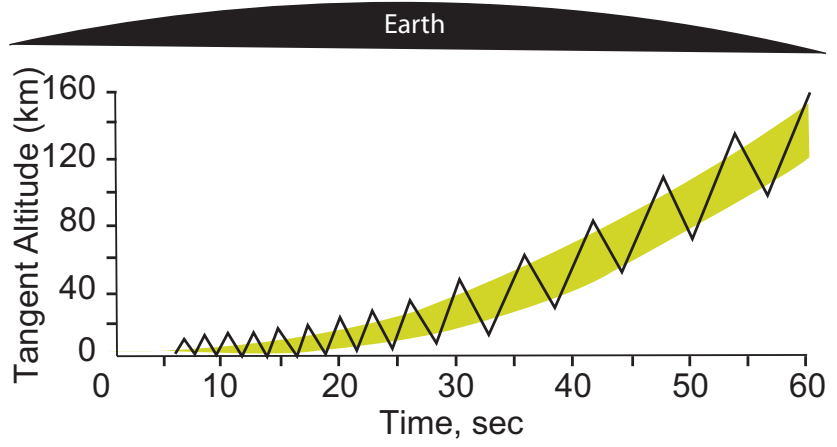

Fig. 1. A depiction of the SAGE II measurement geometry: SAGE II scans across the center solar disk perpendicular to the Earth's surface (above). The figure below shows the pattern of scans for a sunrise event (the location of the Sun is shown in yellow), demonstrating that multiple rays between the instrument and the Sun pass at approximately the same minimum distance from the Earth's surface (the tangent altitude).

\subsection{The interpretation of observations}

Thomason et al. (2008) provide an in-depth discussion of the limitations in the derivation of bulk aerosol properties like surface area density using SAGE II measurements (see also, Thomason et al., 1997a). They showed that there is incomplete information on the details of the underlying aerosol size distribution in the SAGE II extinction coefficient measurements. The nature of the extinction kernels make this relatively obvious particularly when limited to only the 525 and $1020 \mathrm{~nm}$ channels. Figure 2a shows these extinction kernels (in per unit volume of aerosol) for liquid sulfate aerosol at stratospheric temperatures. While there is a clear difference in these kernels, the ratio of these channels, shown in Fig. 2 b, shows that there is only size information for particles less than $0.5 \mu \mathrm{m}$. Since extinction integrates the underlying size distribution across these kernels, we find that the measured ratio of 525 to $1020 \mathrm{~nm}$ aerosol extinction coefficients is close to 1 whenever extinction is dominated by particles larger than $0.5 \mu \mathrm{m}$. This can be relevant to our study since it
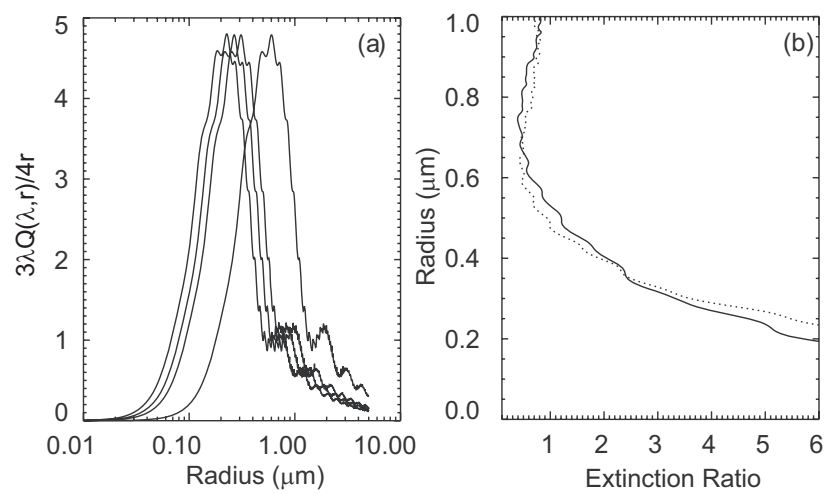

Fig. 2. (a) shows the Mie extinction kernels for the 525 and $1020 \mathrm{~nm}$ SAGE II aerosol extinction coefficient measurements for sulfate aerosol at stratospheric temperatures. (b) shows the relationship between the ratio of the 525 and $1020 \mathrm{~nm}$ extinction kernels and particle radius.

is difficult to conclusively distinguish between large aerosol and cloud. This was commonly observed during the immediate post-Pinatubo period from 1991 through 1993 when stratospheric aerosol levels were more than 100 times larger than in the early 2000s with a concomitant increase in mean particle size. While we generally have based cloud/aerosol discrimination in SAGE II measurements on this extinction ratio, it is important to keep in mind that a small extinction ratio is not a necessary indicator of cloud presence.

Beyond particle size issues, there are additional and substantial ambiguities regarding cloud observations particularly related to optically thin or non-opaque cirrus arising out of the characteristics of the measurement volume. This was demonstrated by Kent et al. (1997) who used data from the Lidar In-space Technology Experiment (LITE) to simulate occultation cloud observations. The ambiguities result from the fact that clouds may not occur at the LOS tangent point but may occur anywhere along the path through the atmosphere and occupy anywhere from the entire path to very short segments of the paths. Kent et al. (1997) demonstrated that either opaque or non-opaque clouds observed by an occultation instrument may occur at or above the altitude in which it is inferred to occur. The difference between the actual height and the inferred height is generally small but can reach as much as $5 \mathrm{~km}$. As a result, cloud events should be interpreted as occurring at or above the apparent altitude. In addition, since the extinction coefficients associated with clouds are assumed to be uniformly spread in the tangent altitude layer, the resulting extinction is a lower limit for extinction by a cloud. Figure 3 shows a schematic of 3 identical clouds occurring at different locations along the LOS: at the tangent altitude and two altitudes well above the tangent altitude. Since the distance traversed through each cloud is also different, the LOS optical depth contributed by the cloud is also different. Since we have no way of knowing where along 


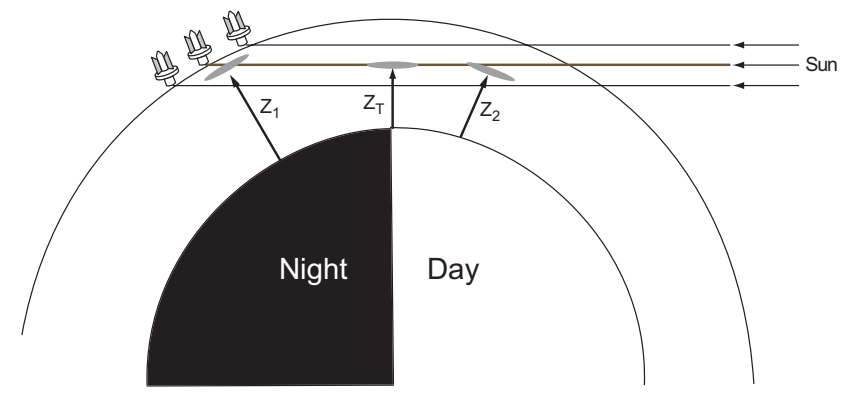

Fig. 3. This figure shows the approximate geometry of the SAGE II observations and demonstrates the different ways in which clouds can manifest themselves in line-of-sight observations. For the center ray in the figure above, clouds at 3 different altitudes $\left(Z_{1}, Z_{2}\right.$, and $Z_{\mathrm{T}}$ ) are all observed for a ray at a single tangent altitude, $Z_{\mathrm{T}}$.

the LOS the clouds occur, SAGE data processing necessarily assumes that each is uniformly distributed in the layer located at the tangent altitude and the reported aerosol extinction coefficient derived for the tangent altitude could be quite different for each cloud position and path. It is also worth noting that in SAGE II versions prior to 6.0 (released in 2000) there was a deficiency in the data-processing algorithms that effectively diffused cloud tops to altitudes well above where they actually occurred (e.g., Wang et al., 1996). This led to an exaggeration in the frequency of cloud/aerosol observation up to and above the tropopause that is greatly reduced in later versions.

Figure 4 shows the density in $1020 \mathrm{~nm}$ extinction and 525 to $1020 \mathrm{~nm}$ extinction ratio space for all SAGE II observations at $18 \mathrm{~km}$ in December/January/February (DJF) for 1999 through 2005 . We see a strong, relatively compact maximum near the $10^{-4} \mathrm{~km}^{-1} 1020 \mathrm{~nm}$ extinction coefficient and 4.5 in extinction ratio (referred to as $k_{\mathrm{a}}$ and $R_{\mathrm{a}}$ below). However, in low numbers we also see a long arm of events stretching from this main core down toward values near $10^{-2} \mathrm{~km}^{-1}$ (near the limit of the observation domain) and extinction ratios of 1 . Following the discussion above, members of this arm of observations are likely to contain either clouds of varying optical density (from relatively dense to aerosol-like opacities), follow varying path lengths through more substantial clouds, or a mix of aerosol-only and cloud-only observations. The observations can be easily modeled using the main aerosol centroid and an "artificial" cloud centroid with extinction ratio of 1 (i.e., large size) and a large "cloud" $1020 \mathrm{~nm}$ extinction coefficient (referred to as $R_{\mathrm{c}}$ and $k_{\mathrm{c}}$ below). We have used a value of $10^{-1} \mathrm{~km}^{-1}$ for the cloud centroid extinction $\left(k_{\mathrm{c}}\right)$ and a value of 1.0 for $R_{\mathrm{c}}$. The model produces an extinction ratio, $R$, for any $1020 \mathrm{~nm}$ extinction coefficient, $k$, between $k_{\mathrm{a}}$ and $k_{\mathrm{c}}$ as a simple mixture of the two centroids, or

$R=\frac{a R_{\mathrm{c}} k_{\mathrm{c}}+(1-a) R_{\mathrm{a}} k_{\mathrm{a}}}{a k_{\mathrm{c}}+(1-a) k_{\mathrm{a}}}$, where $a=\frac{k-k_{\mathrm{a}}}{k_{\mathrm{c}}-k_{\mathrm{a}}}$.

The function is shown overlaying the density plot in Fig. 4.

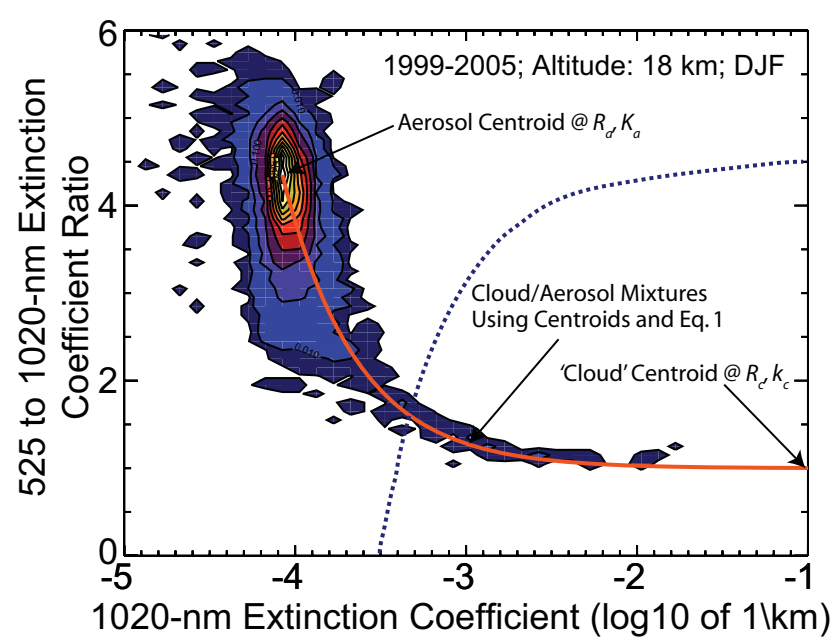

Fig. 4. A typical density function for SAGE II aerosol extinction coefficient observations at $18 \mathrm{~km}$ in DJF for 1999 through 2005. The density is scaled to a value of 1 at the peak value with contours at 0.1 increments $(0.9,0.8$, etc.) and additional contours at $0.05,0.01$, 0.005 and 0.001 . The dotted line shows the approximate position of the "Kent method" cloud/aerosol demarcation. The red line shows the effect of mixing a nominal dense "cloud" with varying fractions of aerosol (values from the centroid of the distribution) using Eq. (1).

As is the case with other seasons and altitudes, the curve does a remarkable job of simulating the observations. The details of the line's location are not strongly modulated by the assumed "artificial" cloud properties except in that they are spectrally white and optically dense relative to the background aerosol. The line would be nearly identical for any $k_{\mathrm{c}}$ value over $10^{-3} \mathrm{~km}^{-1}$. The robustness of this fit suggests that these observations are likely to represent mixtures of background aerosol with some cloud-like component. This does not imply that they are mixed uniformly in a volume of air but mixed by geometry along the line of sight with segments of aerosol and cloud. It also suggests that terminologies used on occasion like "optically thin cirrus" or "sub-visual cirrus" may be misleading in as much as they are applied to SAGE II observations and that it may be better to refer to these observations as cloud/aerosol mixtures as originally recognized by Kent et al. (1993, 1998). As discussed above, how the atmosphere and the SAGE II observations construct this mixture is not readily determinable from the data.

\section{Distinguishing between aerosol and clouds in SAGE II observations}

The focus of this paper is on aerosol in the UTLS. To facilitate this study, we were required to identify (for the purposes of removal) measurements that exhibit cloud-like properties. Our original intent was to employ the method pioneered by Kent et al. (1993) which is included in operational SAGE II 
data product. While we have found it to be reasonably effective, based on past experiences, we felt that at least some cloud-like observations were missed by this algorithm. As a result, we have explored a fusion of the Kent method with a method based on the polar stratospheric cloud discrimination scheme developed by Pitts et al. (2009) for use with the CALIPSO data set.

\subsection{The Kent method}

The cloud/aerosol discrimination method developed by Kent et al. (1993) (called the Kent method below) is based on the observed SAGE II 525 and $1020 \mathrm{~nm}$ aerosol extinction coefficient distribution. These authors also recognized that many "thin" clouds were in fact cloud/aerosol mixtures and that the transition from pure aerosol measurements to cloud/aerosol mixtures is a continuum rather than a discrete step. The method is predicated on the assumption that cloud/aerosol mixtures have an extinction ratio approaching 1 (large particle size) whereas aerosol typically has an extinction between 2 and 5. The dividing line between the two types is expressed as a simple linear equation in the form

$k_{525}=m\left(k-k_{i}\right)$,

where $m$ is slope of the line that divides aerosol and cloud/aerosol mixtures in $525 \mathrm{vs.} 1020 \mathrm{~nm}$ aerosol extinction coefficient space and $k_{i}$ is the intercept of this line on the $1020 \mathrm{~nm}$ extinction coefficient axis. While a detailed discussion of the Kent method is beyond the scope of this paper, the source of the values derived for the parameters is relatively easy to understand. The parameter $m$ is roughly the 525 to $1020 \mathrm{~nm}$ extinction coefficient ratio that is most characteristic of the primary aerosol centroid. For the distribution shown in Fig. 4 , the equivalent value of $m$ would be $\sim 4.5$. The value for $k_{i}$, along with the slope, helps to define the positive (high extinction) edge of the main aerosol centroid.

Values of $m$ and $k_{i}$ are computed in as a function of altitude, latitude, season and year. These coefficients have not been formally archived, but they are available from the authors of this paper. The Kent method was used in the SAGE II version 6.2 data set as one of the data quality flags. In the variable space used in Fig. 4, the Kent method is expressed as

$R=m-\frac{m k_{i}}{k}$.

Figure 4 shows an example of the division between aerosol and cloud/aerosol mixtures based on the Kent method (dotted line). Since this plot includes data from multiple years and latitudes, the curve (for DJF 2002) is not completely matched with the data plot. However, the results for the Kent method fit do not change significantly with latitude, and this curve is typical of those found between 1999 and 2005. Generally, it shows that the Kent method is doing a good job separating aerosol from cloud/aerosol mixtures, though it seems likely that some aerosol/cloud mixtures remain in the aerosol category in the region where extinction is near $2 \times 10^{-4} \mathrm{~km}^{-1}$ and the extinction ratio is near 2. Geographically, we find that many of these transitional cloud/aerosol mixtures occur, not surprisingly, in the tropics. This matches past experiences where efforts to remove cloud impacts from the aerosol data left enough mixtures in the data set that it was better to use median statistics (with mixed results) than averaging in order to minimize residual cloud impacts (e.g., Thomason et al., 1997b). This effect is one of the primary motivations for producing a modified form of the Kent method for this analysis.

\subsection{The new method}

The modifications to the Kent method involve using a space change from 525 vs. $1020 \mathrm{~nm}$ aerosol extinction space to the extinction ratio vs. $1020 \mathrm{~nm}$ aerosol extinction space that is analogous to the polar stratospheric cloud identification scheme developed by Pitts et al. (2009). In their approach, aerosol depolarization ratio and backscatter ratio are used with some straightforward statistical modeling to separate aerosol from polar stratospheric clouds of various compositions. Following their approach, we first distinguish between the primary aerosol centroid and enhanced extinction using a probably density function of aerosol-extinctionexcluding measurements where the 525 to $1020 \mathrm{~nm}$ aerosol extinction coefficient ratio is less than 2. An example of this distribution is shown in Fig. 5. The boundary between the aerosol primary aerosol and enhanced aerosol, $k_{\mathrm{o}}$, is defined as $k_{\mathrm{a}}+3 \Delta k_{\mathrm{a}}$, where $\Delta k_{\mathrm{a}}$ is the median absolute deviation of aerosol extinction coefficient with extinction ratio greater than 2 from $k_{\mathrm{a}}$. We find that the factor of 3 is reasonably robust for above $12 \mathrm{~km}$ but should be decreased below $12 \mathrm{~km}$ to values as small as 1.5. The factor of 3 was selected based on an evaluation of where the cloud "tail" originated and, as such, is empirical, but we are generally pleased with the overall results. Nonetheless, the details of separating cloud and aerosol is steadfastly subjective. Within the UTLS and stratosphere, the distribution is generally quite narrow; more than $95 \%$ of points with extinction ratios greater than 2 lie below $k_{\mathrm{o}}$. As will be shown below, this fraction decreases at lower altitudes where higher extinction but still optically small particles (extinction ratio greater than 2) are more common. The area greater than $k_{\mathrm{o}}$ is subdivided using Eq. (1) except where the ratio, $R$, is offset from the aerosol centroid and notional cloud path by an empirically selected value $\delta$, or

$R=\frac{a R_{\mathrm{c}} k_{\mathrm{c}}+(1 .-a) R_{\mathrm{a}} k_{\mathrm{a}}}{a k_{\mathrm{c}}+(1 .-a) k_{\mathrm{a}}}+\delta$.

Generally, we find that values for $\delta$ between 0.2 and 0.5 are adequate to delineate between the upper limit of cloud/aerosol mixtures and that the resulting aerosol analyses are not sensitive to the value chosen (though some inferences of cloud properties and occurrence can be significantly 


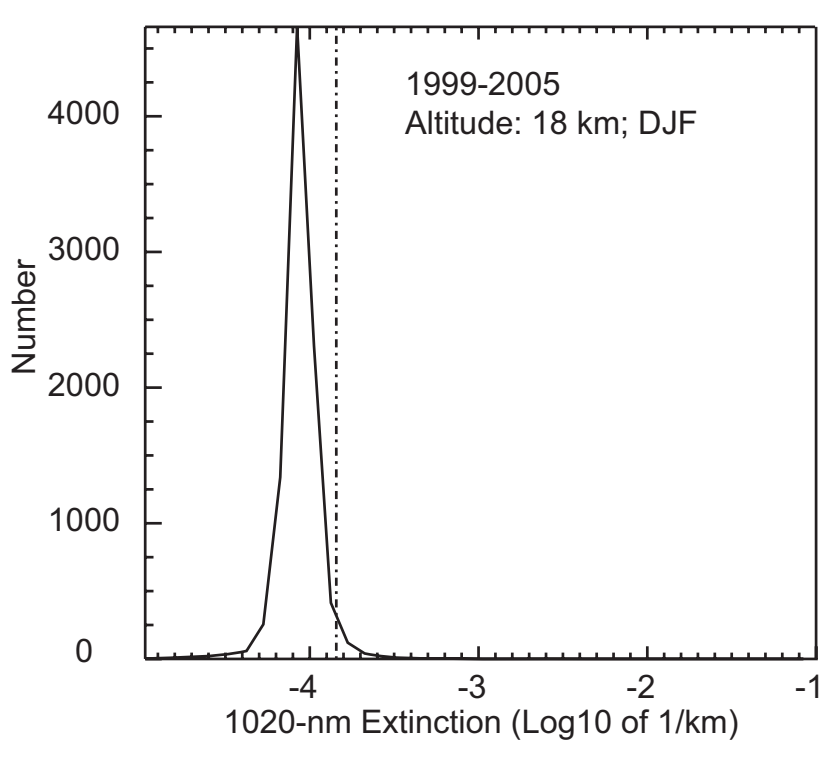

Fig. 5. The density of SAGE II aerosol extinction coefficient measurements at $18 \mathrm{~km}$ in DJF for $1999-2005$ for 525 to $1020 \mathrm{~nm}$ aerosol extinction coefficient ratio greater than 2 . The dash-dot line is the location of the separation of primary aerosol from enhanced aerosol and cloud/aerosol mixtures.

affected). In practice, we use a fixed value of 0.4. The purpose of the offset is to account for the spread in the aerosol/cloud mixture tail observed in Fig. 4. For higher extinctions, we would expect ratios very close to 1.0 , but, due primarily to measurement noise in the $525 \mathrm{~nm}$ extinction coefficient, we observe a spread of a few tenths in the extinction ratio which needs to be accommodated. Observations with extinction coefficient greater than $k_{\mathrm{o}}$ and extinction ratio lying above the line $R$ are designated as "enhanced aerosol" and most commonly occur below $10 \mathrm{~km}$ and at high northern latitudes in spring and summer. No matter how large the extinction these point exhibit, we do not believe that measurements lying within the enhanced aerosol area are compatible with cloud observations, and their observed morphology (when and where they occur) is also consistent with being aerosol. Observations with extinction coefficient greater than $k_{\mathrm{o}}$ but with extinction ratio less than $R$ are considered cloud/aerosol mixtures and for the purposes of the analysis below are excluded from further analysis. These boundaries are shown in Fig. 6. Note that there is a wedge-shaped region in this figure (denoted by the letter $\mathrm{W}$ ) where classification of observations is problematic. In practice, we count these as cloud/aerosol mixtures and exclude them from aerosol analyses since they have little impact on the products shown below. On the other hand, other statistics such as cloud frequency, particularly at altitudes above $15 \mathrm{~km}$, are impacted by whether we consider these events to be cloud observations. We know of no practical way to make this judgment. Given the dependence on having both 525 and $1020 \mathrm{~nm}$ extinction coefficients, this analysis can only be car-

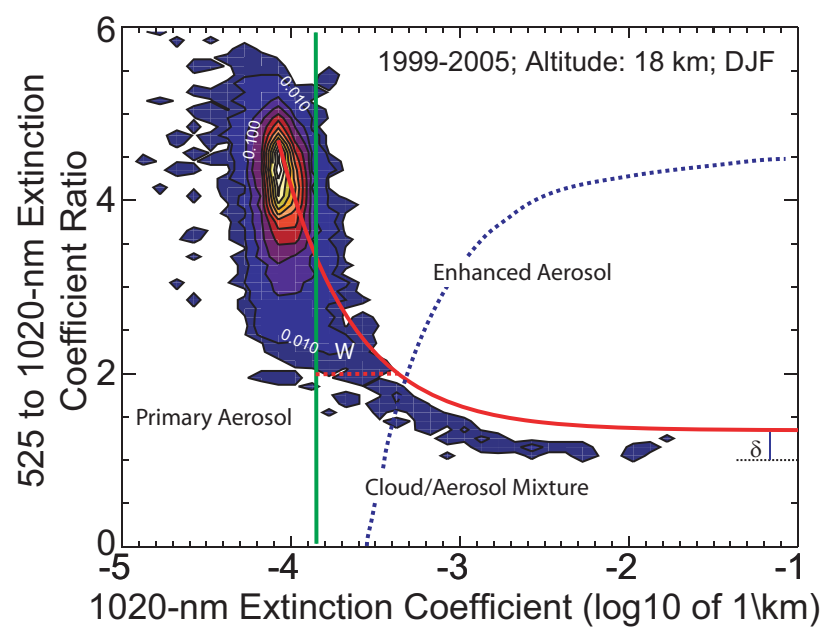

Fig. 6. A typical density function for SAGE II aerosol extinction coefficient observations at $18 \mathrm{~km}$ in DJF for 1999 through 2005. The density is scaled to a value of 1 at the peak value with contours at 0.1 increments $(0.9,0.8$, etc.) and additional contours at 0.05 , $0.01,0.005$ and 0.001 . The dotted line shows the approximate position of the "Kent method" cloud aerosol demarcation. The green line shows the separation of primary aerosol (left) from enhanced aerosol and clouds, while the red line (when right of the green line) shows the separation of enhanced aerosol and cloud/aerosol mixtures. This line is determined by the location of the aerosol centroid, the nominal cloud centroid and Eq. (1) offset upward in extinction ratio by $\delta$ (in this case, 0.4 ). The area denoted by a "W" is the wedge region in which the distinction between aerosol and cloud/aerosol mixtures is particularly ambiguous.

ried out down to altitudes of $5 \mathrm{~km}$ (the last altitude at which the $525 \mathrm{~nm}$ extinction coefficient is routinely reported); however, the quality of this measurement is suspect at $5 \mathrm{~km}$ and practically the analysis can be carried out no lower than $6 \mathrm{~km}$, while $8 \mathrm{~km}$ would be a more circumspect cutoff altitude. Due to uncertainty in the fraction of clouds occupying the measurement volume, extinction coefficient values for observations identified as aerosol/cloud mixtures should not be taken as representative of cloud extinction values under any circumstance.

During the bulk of the "clean" period of interest in the following discussion, the parameters going into the cloud/aerosol separation are not strongly dependent on season or latitude. There is significant dependence on altitude particularly below $10 \mathrm{~km}$ as aerosol levels even for the nominal primary aerosol increases. Figure 7 shows the altitude dependence of two key parameters, $R_{\mathrm{a}}$ and $k_{\mathrm{o}}$, for DJF between 1999 and 2005. The figure highlights the difficulty in separating clouds and aerosol in the lowest few kilometers of the SAGE II data set as the extinction ratio decreases rapidly below $8 \mathrm{~km}$ and is nearly 1.5 by $6 \mathrm{~km}$. Since the cloud/aerosol separation is strongly dependent on the extinction ratio, the lack of contrast between aerosol extinction ratio and cloud ratio is a limiting factor. Given the challenges to 
separating aerosol from aerosol/cloud mixtures, validation of this methodology would be highly valuable. Unfortunately, we find the large measurement volume associated with solar occultation measurements (Thomason et al., 2003) and the inhomogeneity of clouds on this scale make validation of this method using ground-based or even aircraft-based measurements extremely difficult, and adequate data for this study does not currently exist.

In practice, we find that the new method yields results similar to the Kent method above $15 \mathrm{~km}$ and away from the tropics. However, within the tropics and at lower altitudes, it is clear (and can easily be surmised from Fig. 6) that some measurements that should be classified as cloud/aerosol mixtures are erroneously being classified as aerosol. Since these classification differences preferentially occur at low latitudes, the impact of identifying these events as either cloud or aerosol has a significant impact on our following analysis. In addition, we find that enhanced aerosol, when it occurs, is often misclassified as cloud/aerosol mixture by the Kent method. In general, we find that the new approach is less likely to confuse aerosol and cloud/aerosol measurements particularly in the clean period for the late 1990s through the end of the observations in 2005. It is not clear that either method works particularly well (or that it is possible for them to do so) in the heavily volcanic period in the early 1990s where the extinction ratio for aerosol is close to 1 . It is theoretically possible to continue analysis with only the $1020 \mathrm{~nm}$ extinction coefficient, for instance, by using $k_{\mathrm{o}}$ as the sole discriminator between aerosol and cloud. We find that it is inevitable that enhanced aerosol will be counted as cloud with the potential for substantially confusing any analysis of aerosol and/or cloud properties. For this reason, we find the use of a single wavelength method at any altitude to be of questionable value.

\section{Observations of the Asian tropopause aerosol layer}

While highly variable in intensity from year to year, CALIPSO observations have consistently shown the presence of an aerosol enhancement within the Asian monsoon anticyclone. This layer appears as early as May and persists as late as September but is primarily a JJA phenomenon; it is correlated with the Asian monsoon anticyclone (Vernier et al., 2011b) and is also well correlated with enhancements of $\mathrm{CO}$ and $\mathrm{HNO}_{3}$ as reported by Randel et al. (2010). The spatial extent was observed to lie between 14 and $18 \mathrm{~km}$ and between roughly 15 and $35^{\circ} \mathrm{N}$ and 0 and $150^{\circ} \mathrm{E}$. While SAGE II observations extend for over $20 \mathrm{yr}$, no previous analysis of SAGE II over southern Asia has suggested the presence of the ATAL aerosol. Part of the reason for this is due to the performance of the Kent method that led to mixing of purely aerosol observations with cloud/aerosol mixture observations. This added a non-aerosol enhancement to aerosol extinction coefficient depictions throughout the ATAL alti-
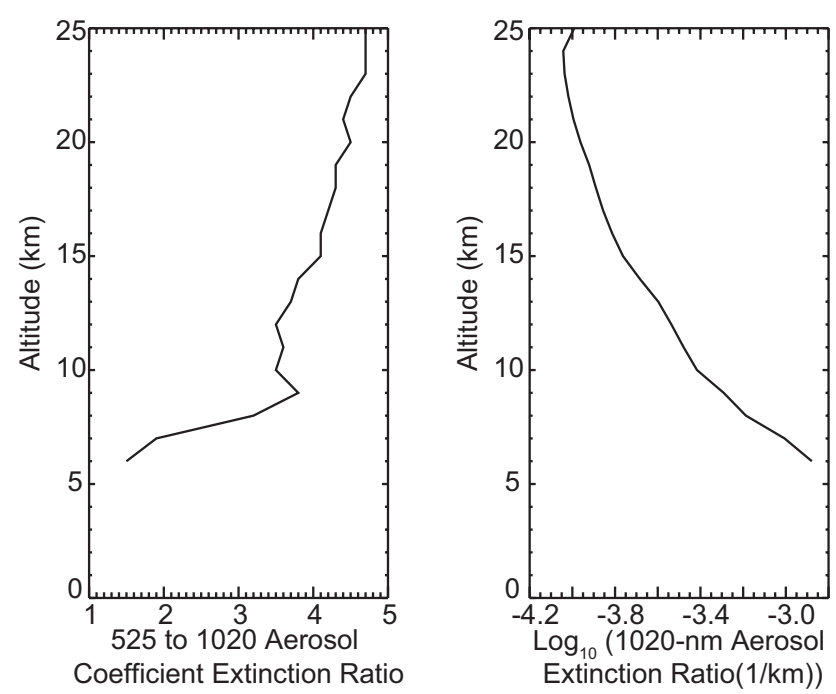

Fig. 7. The vertical profiles of aerosol coefficient extinction ratio centroid, $R_{\mathrm{a}}$, (left) and the extinction coefficient value for the separation of primary aerosol from enhanced aerosol and clouds, $k_{\mathrm{O}}$, (right) for DJF between 1999 through 2005.

tude region and masked its presence. The development of the new cloud/aerosol discrimination method has greatly reduced this problem. The second issue is that, particularly after 2000, the density of observations by SAGE II over Asia (as well as over Africa and South America) are so sparse as to make detailed analyses extremely challenging. The analyses of CALIPSO data shown in Vernier et al. (2011b) are made in 16-day, $1^{\circ}$ latitude $\times 2^{\circ}$ longitude $\times 200 \mathrm{~m}$ resolution. While this yields a somewhat noisy analysis, the presence of the ATAL feature is clearly visible in July-August observations throughout the CALIPSO mission (2006-present). Unfortunately, SAGE II simply does not make sufficient measurements to approach this resolution in any meaningful way. Instead the following analyses are based on seasonal statistics (by months) with a spatial resolution of $10^{\circ}$ latitude $\times$ $24^{\circ}$ longitude $\times 0.5 \mathrm{~km}$. We use medians to select the value reported in each bin. In individual years, mean and median statistics are very similar. In the multiyear analysis, some episodic events, particularly in 2003, have a large impact on the analysis, and the median analysis is generally more representation of the period as a whole. For single-year analyses prior to 2000, depending on the specific year and season, the analysis resolution puts between 20 and 60 points into each bin with some high-latitude bins completely without data (i.e., the winter hemisphere). While it is possible for the bin values to be controlled by outliers, by-hand inspection of many grid boxes, particularly of the ATAL feature, indicate that the final analyses are representative. For individual years after 1999, the analyses have a number of unpopulated or minimally populated bins in the subtropics, making detailed analyses of ATAL considerably more difficult. 

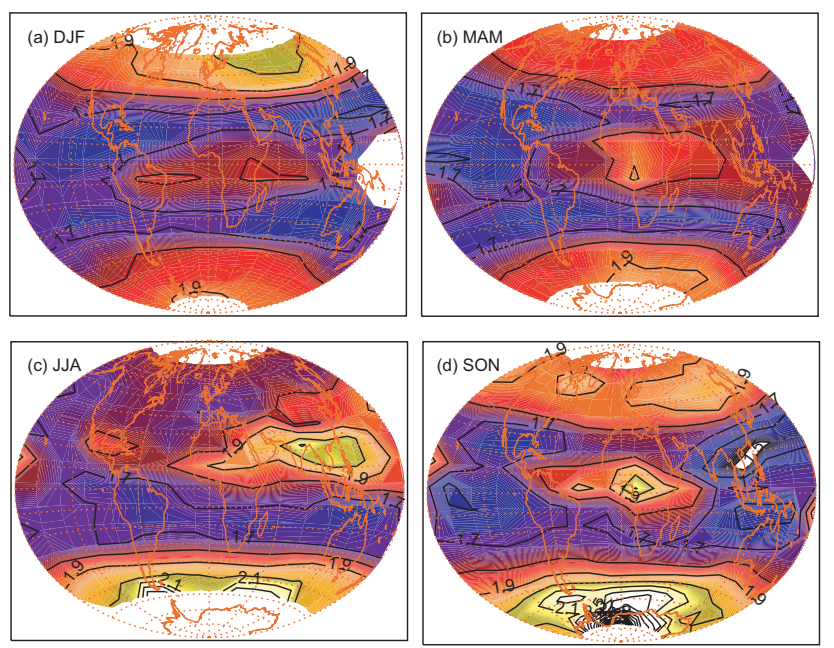

Fig. 8. Seasonal depiction of $1020 \mathrm{~nm}$ aerosol extinction coefficient ratio (relative to molecular) for 1999-2005 at $16 \mathrm{~km}$. Contour levels are drawn at 0.1 increments from 1.5 to 2.2 .

Figure 8 shows the $1020 \mathrm{~nm}$ aerosol/molecular extinction ratio $(1020 \mathrm{~nm}$ aerosol extinction coefficient divided by molecular extinction coefficient plus 1) for $16 \mathrm{~km}$ at the grid resolution shown above for DJF (December/January/February), MAM (March/April/May), JJA (June/July/August) and SON (September/October/November) for the combined $7 \mathrm{yr}$ between 1999 and 2005. We use the extinction ratio in this and subsequent plots since it is as close to an aerosol "mixing ratio" as SAGE II measurements permit and as such is less sensitive to altitude changes and is generally easier to infer relative changes from than extinction coefficient depictions. In these plots, we found a general increase toward higher latitudes generally associated with crossing from the upper troposphere at low latitudes to the lower stratosphere at higher latitudes. SAGE II observations routinely show the tropical upper troposphere to have lower aerosol extinction coefficient levels than the overlying stratosphere. In addition, the influence of polar stratospheric clouds has some influence in the winter/spring hemisphere in the Southern Hemisphere. Most PSC types (supercooled ternary solution (STS) and nitric acid trihydride/STS mixtures) are generally identified as "aerosol" by the aerosol/cloud separation method (whereas ice PSCs are generally identified as clouds) and are included in this analysis as aerosol. At low latitudes, we observe an enhancement of aerosol near the equator that is spatially associated with Africa and to a lesser extent South America. Since this feature is strongest in September/October/November (SON) when biomass burning is most intense in equatorial Africa (Roberts et al., 2009), it is probably associated with that phenomena. Since a corresponding feature does not appear in the CALIPSO data where cloud/aerosol discrimination is based on depolarization (effectively a solid/liquid difference), it is possible
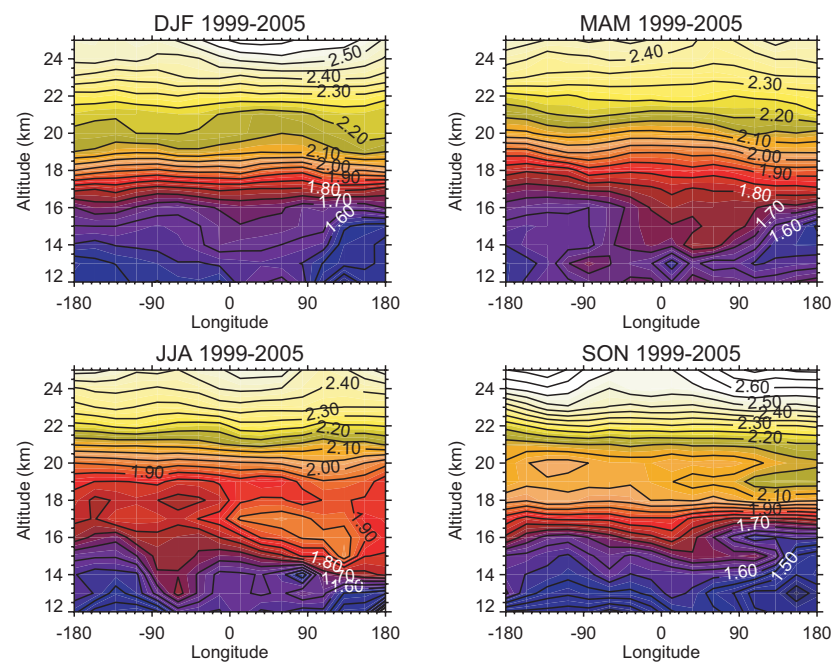

Fig. 9. Seasonal depictions of median aerosol extinction coefficient ratio (relative to molecular) for SAGE II observations between 1999 and 2005 between 15 and $45^{\circ} \mathrm{N}$. Contours are drawn every 0.05 from 1.4 to 3.0 .

that this enhancement consists of small, solid particles unlike ATAL, which is likely to be small liquid aerosol. In all seasons, except JJA, the subtropics and mid-latitudes are found to be at low aerosol/molecular extinction coefficient ratio values. In JJA, however, we observe the largest the extinction coefficient values, outside of regions affected by PSC presence, over southern Asia, particularly India and mainland Southeast Asia. In this area, the aerosol extinction coefficient increases from between 1.5 and 1.75 during the other 3 seasons to over 2 , or a 50 to $100 \%$ increase in the aerosol component. The magnitude of the increase and geographic scope of this feature is extremely compatible with the ATAL feature found in the CALIPSO analysis by Vernier et al. (2011b) but extends further east $\left(\sim 180^{\circ} \mathrm{E}\right)$ than the layer found by CALIPSO analysis $\left(\sim 150^{\circ} \mathrm{E}\right)$. The expanded eastward extent may be partly related to the reduced horizontal resolution (relative to CALIPSO) of the SAGE II analysis.

Figure 9 shows the seasonal vertical cross section of the aerosol/molecular extinction ratio for JJA for latitudes between 15 and $45^{\circ} \mathrm{N}$ as a function of longitude and altitude for the $7 \mathrm{yr}$ from 1999 through 2005. During the non-NH summer months, we observe little evidence of a discrete aerosol layer between 14 and $18 \mathrm{~km}$ (ATAL altitudes) for any longitude or altitude, though there is a weak enhancement in the Eastern Hemisphere in MAM and SON which may reflect the May-to-September lifetime of ATAL found by Vernier et al. (2011b). Generally, the aerosol/molecular extinction coefficient ratio mostly increases monotonically upward from $12 \mathrm{~km}$ across the tropopause and into the stratosphere where the highest ratios are observed. In this analysis, episodic minor volcanic events do not have a substantial effect except in 
the SON analysis around $20 \mathrm{~km}$ which reflects an influence by the September 2002 Ruang eruption. On the other hand, a significant aerosol enhancement in the JJA analysis in the same altitude range as ATAL is observed by CALIPSO beginning in 2006. The layer extends from about 0 to $160^{\circ} \mathrm{E}$, is centered between 14 and $18 \mathrm{~km}$ and thus has a similar spatial extent as that observed by CALIPSO. The entire latitude band shows enhanced aerosol relative to the other seasons, and there is a suggestion of an ATAL-like feature near $90^{\circ} \mathrm{W}$ that could be associated with the North American monsoon, but it is much weaker and of significantly smaller extent. Overall, we find that the 1999-2005 analysis supports the presence of the ATAL in this period.

Vernier et al. (2011b) found that the ATAL was highly variable from year to year even beyond the effect of volcanic activity. Similar analyses by SAGE II are difficult and potentially noisy, but the potential to extend the ATAL record as far back as the 1980s is desirable. Unfortunately we find that the stratospheric loading and its subsequent impact on the upper troposphere effectively eliminate a number of years due to high in situ levels in the UTLS that overwhelm ATAL levels observed in the 2000s. As a result, we are only able to produce useful depictions for a few years in the late 1980s and then 1996 through 1999 (until the reduction to a 50\% data rate). SAGE II operations were terminated in August 2005 and very little low latitude data is available for that JJA period. Figure 10 shows the single-year analyses for JJA for 1989, 1997, 1998 and 1999 (the last year in which SAGE II operated using a complete duty cycle during JJA). We observe that both 1989 and 1997 do not show any evidence of an aerosol enhancement at ATAL longitudes and, in fact, tend to support the 14 to $18 \mathrm{~km}$ region as a minimum in aerosol loading. Analyses for 1990 and 1996, not shown, also do not suggest the presence of the ATAL. In fact, the entire 14 to $18 \mathrm{~km}$ region in the 1999-2005 analysis (Fig. 9c) shows a considerably higher aerosol extinction coefficient than any of the 4 yr shown in Fig. 10. The years 1998 and 1999 do show some enhancement in the areas over Asia. In 1999, there is a distinct layer near $18 \mathrm{~km}$; the aerosol seems to be associated with recent volcanic activity or other episodic events (e.g., eruption of Mayon, Philippines, June 1999) and persists into 2000, which is unlike the short lifetime of the ATAL-related aerosol. This event may be the source of aerosol reported by Tobo et al. (2007) and Kim et al. (2003). Similarly, the feature in 1998 at $17 \mathrm{~km}$ and near $90^{\circ} \mathrm{E}$ is also the product of an episodic event (possibly material from eruptions by Soufriere Hills or Colima) as it continued to intensify into SON before dissipating by DJF, again unlike the characteristic ATAL behavior. There may be some hints of an ATAL feature in 1999 between 14 and $17 \mathrm{~km}$ near $100^{\circ} \mathrm{E}$, but they are not very compelling.

While for individual years 2000 and later easily interpretable plots like those in Fig. 10 are difficult to produce, it is possible to infer the presence of ATAL using extinction coefficient frequency density functions similar to the
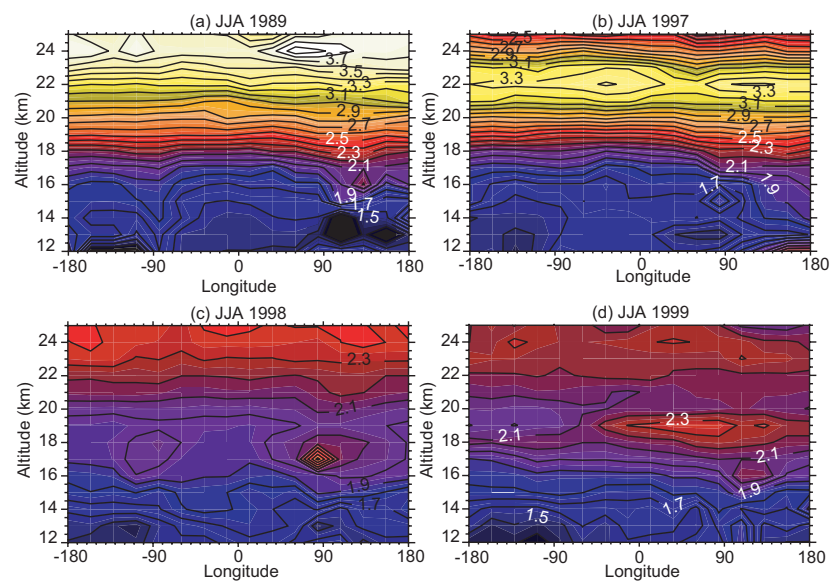

Fig. 10. JJA depictions of median aerosol extinction coefficient ratio (relative to molecular) for SAGE II observations between 15 and 45 ${ }^{\circ} \mathrm{N}$ for individual years: 1989 (a), 1997 (b), 1998 (c), and 1999 (d). Contours are drawn every 0.1 from 1.4 to 4.0 .

backscatter ratio frequency density plots used in Vernier et al. (2011b). In that paper, the authors found that the backscatter ratio density function within the main ATAL region increased by about a factor of two between DJF and JJA without an appreciable change in the shape of the distribution in 2006 through 2008. The outlier in that analysis was the heavily volcanic year 2009 which showed the largest increase and a significant perturbation to the shape of the density function. In Fig. 11, we show the density functions of the $1020 \mathrm{~nm}$ aerosol extinction within the ATAL region and, in Fig. 12, an associated scatter plot of $1020 \mathrm{~nm}$ extinction coefficient versus the 525 to $1020 \mathrm{~nm}$ aerosol coefficient ratio for a selection of years between 1989 and 2005, focusing mostly on the late 1990s through the end of the SAGE II record. In this case, we follow the analysis by Vernier et al. (2011b) and use all SAGE II observations between 5 and $105^{\circ} \mathrm{E}, 15$ and $45^{\circ} \mathrm{N}$, and 15 to $17 \mathrm{~km}$. In each plot we show both the MAM and JJA analyses. In Fig. 11, the density functions show an increase from MAM to DJF in extinction coefficient between 1999 and 2004. Some of these years show increases on par with those found (for backscatter ratio) in the CALIPSO analysis $(\sim 2)$, but a few show much larger increases. Similar to 1999 , it seems likely that at least 2003 is the result of an episodic event though we cannot identify its source. Unlike most ATAL features which dissipate rapidly in SON, the 2003 feature strengthens into the fall though the observations may suggest some trapping of material within the Asian monsoonal circulation (Bourassa et al., 2012). One concern with the analysis would be that clouds are still slipping by the analysis and artificially creating an aerosol feature. The scatter plots shown in Fig. 12 clearly demonstrate that this is not the case. While the aerosol points are essentially the same between seasons in 1989, 1990, and 1997, there is a clear shift in the position of the core aerosol in the plots for 1999 

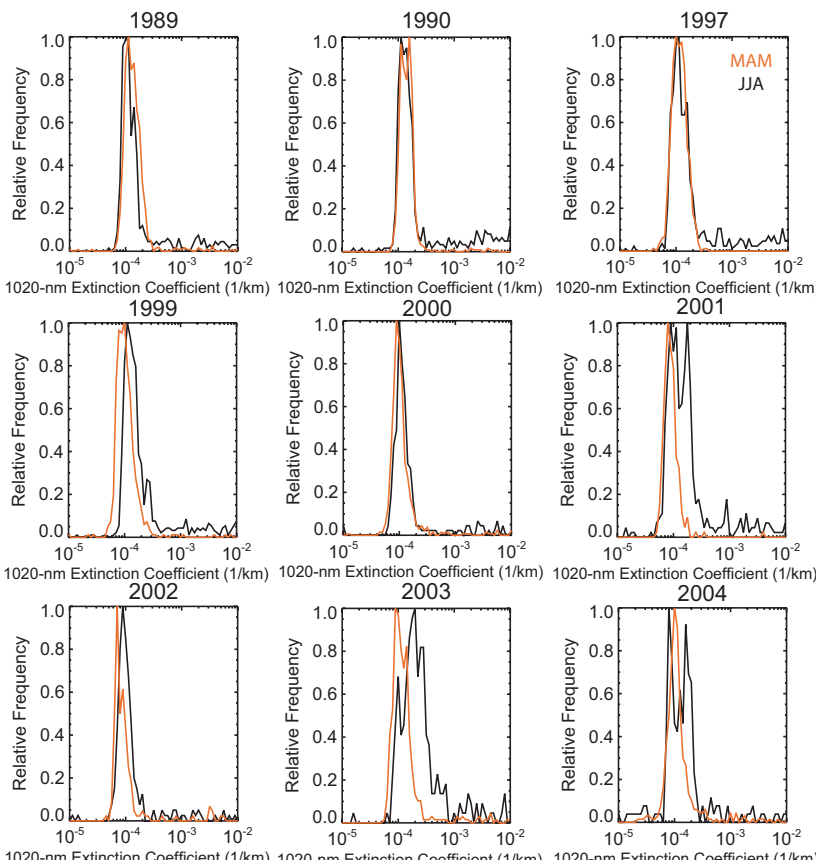

Fig. 11. Aerosol $1020 \mathrm{~nm}$ extinction coefficient density as a function of extinction coefficient for selected years between 1989 and 2005. Data from MAM is shown in red while data for JJA is shown in black.

through 2005. While there are obviously many more cloud events in JJA than MAM, the primary shift in extinction coefficient occurs for aerosol extinction ratios above 2, which are almost certainly aerosol. It is worth noting that prior to 1999, the aerosol extinction coefficient between MAM and JJA was never observed to increase within the ATAL zone. Some of this is due to the long recovery from Pinatubo particularly immediately after the eruption; however nothing in the SAGE II data set suggests an ATAL feature before 1999. Unlike from 1997 and earlier, extinction density distributions starting in 1999 are always shifted toward larger extinction values with the exception of 2005 (not shown) wherein August data is missing. Some of these functions have multiple peaks; the reason for this change in shape is not clear and it is unlike the behavior exhibited in the CALIPSO analyses. Overall, we observe behavior in the SAGE II data that is very similar to CALIPSO ATAL observations in 1999, 2000 and 2002. The behavior is similar to CALIPSO, except for a change in shape, in 2001 and 2004. The SAGE II analysis does not observe an ATAL-like aerosol enhancement in 2005 (when August data is unavailable) or in JJA 2003 where the enhancement is much larger than any SAGE II or CALIPSO ATAL enhancement. Based on this analysis, we conclude that SAGE II suggests that the ATAL aerosol feature did not exist prior to the late 1990s but that there is sufficient evidence from the SAGE II data to indicate its presence from 1999 onwards.
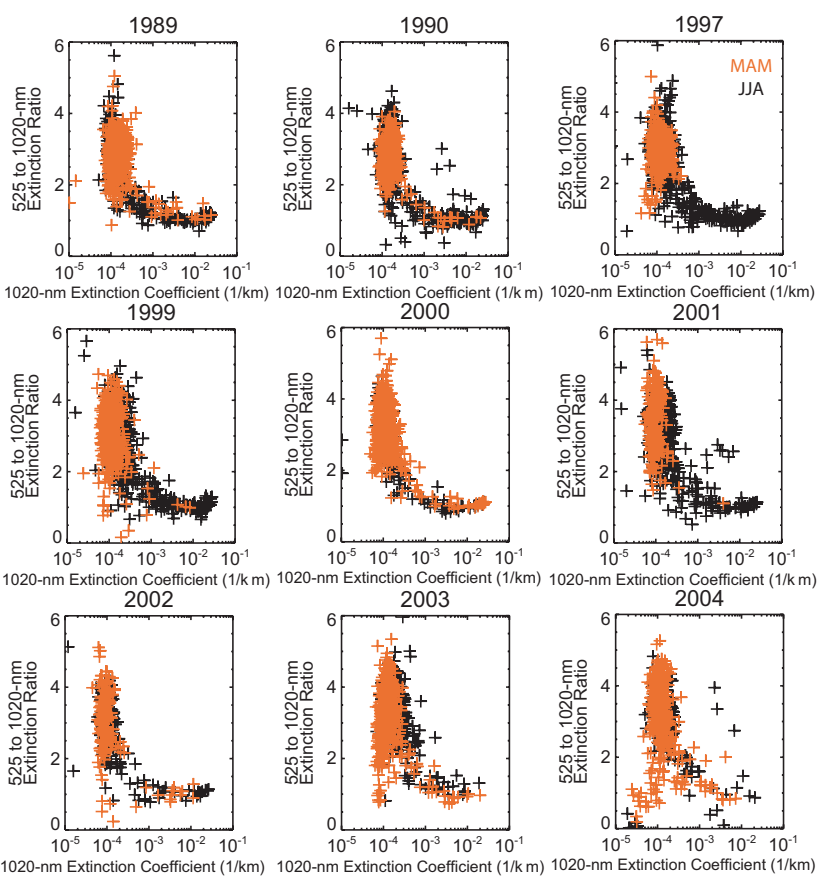

Fig. 12. Scatter plots of SAGE II measure 525-1020 nm aerosol extinction coefficient ratio to aerosol $1020 \mathrm{~nm}$ extinction coefficient density for selected years between 1989 and 2005. Data from MAM is shown in red while data for JJA is shown in black.

\section{Conclusions}

An improved method for identifying SAGE II observation in the upper troposphere influenced by the presence of clouds has been developed. The new method significantly reduced the influence of clouds on the inference of aerosol properties in the UTLS. We have applied this new method to analyses of aerosol in the Northern Hemisphere subtropics where observations by CALIPSO have revealed enhanced aerosol associated with Asian monsoon. This enhancement, called the Asian Tropopause Aerosol Layer (ATAL), appears in every year of the CALIPSO lifetime and is clearly present in the 1999-2005 SAGE II analysis. Individual year analysis shows no indication of the ATAL in the SAGE II data prior to 1998. During the period of 1999 through 2005, the SAGE II analysis is generally consistent with the existence of an ATAL-like feature. Based on this analysis, we conclude that ATAL is a relatively recent phenomenon (1999 and later).

Edited by: G. Stiller 


\section{References}

Bourassa, A. E., Robock, A., Randel, W., Deshler, T., Rieger, L., Nicholas, D., Llewellyn, L., and Degenstein, D.: Large Volcanic Aerosol Load in the Stratosphere Linked to Asian Monsoon Transport, Science, 337, 78-81, doi:10.1126/science.1219371, 2012.

Kent, G. S., Winker, D. M., Osborn, M. T., McCormick, M. P., and Skeens, K. M.: A model for the separation of cloud and aerosol in SAGE II occultation data, J. Geophys. Res., 98, 20725-20735, 1993.

Kent, G. S., Winker, D. M., Vaughan, M. A., Wang, P.-H., and Skeen, K. M.: Simulation of Stratospheric Aerosol and Gas Experiment (SAGE) II cloud measurements using airborne lidar data, J. Geophys. Res., 102, 21795-21807, 1997.

Kent, G. S., Trepte, C. R., and Lucker, P. L., Long-term Stratospheric Aerosol and Gas Experiment I and II measurements of upper tropospheric aerosol extinction, J. Geophys. Res., 103, 28863-28874, 1998.

Kim, Y. S., Shibata, T., Iwasaka, Y., Shi, G., Zhou, X., Tamura, K., and Ohashi, T.: Enhancements of aerosols near the cold tropopause in summer over Tibetan Plateau: Lidar and balloon borne measurements in 1999 at Lhasa, Tibet, China, Proc. SPIE, 4893, 496-503, doi:10.1117/12.466090, 2003.

Pitts, M. C., Poole, L. R., and Thomason, L. W.: CALIPSO polar stratospheric cloud observations: second-generation detection algorithm and composition discrimination, Atmos. Chem. Phys., 9, 7577-7589, doi:10.5194/acp-9-7577-2009, 2009.

Randel, W. J., Park, M., Emmons, L., Kinnison, D., Bernath, P., Walker, K. A., Boone, C., and Pumphrey, H.: Asian monsoon transport of pollution to the stratosphere, Science, 328, 611-613, doi:10.1126/science.1182274, 2010.

Roberts, G., Wooster, M. J., and Lagoudakis, E.: Annual and diurnal african biomass burning temporal dynamics, Biogeosciences, 6 , 849-866, doi:10.5194/bg-6-849-2009, 2009.

SPARC: Assessment of Stratospheric Aerosol Properties (ASAP), SPARC Report No. 4, WCRP-124, WMO/TD-No. 1295, February 2006, edited by: Thomason, L. and Peter, Th., 2006.

Thomason, L. W., Kent, G. S., Trepte, C. R., and Poole, L. R.: A comparison of the stratospheric aerosol background periods of 1979 and 1989-1991, J. Geophys. Res., 102, 3611-3616, 1997a.
Thomason, L. W., Poole, L. R., and Deshler, T. R.: A global climatology of stratospheric aerosol surface area density as deduced from SAGE II: 1984-1994, J. Geophys. Res., 102, 8967-8976, $1997 b$.

Thomason, L. W., Herber, A. B., Yamanouchi, T., and Sato, K.: Arctic Study on Tropospheric Aerosol and Radiation: Comparison of tropospheric aerosol extinction profiles measured by airborne photometer and SAGE II, Geophys. Res. Lett., 30, 1328-1331, doi:10.1029/2002GL016453, 2003.

Thomason, L. W., Burton, S. P., Luo, B.-P., and Peter, T.: SAGE II measurements of stratospheric aerosol properties at non-volcanic levels, Atmos. Chem. Phys., 8, 983-995, doi:10.5194/acp-8-9832008, 2008.

Tobo, Y., Iwasaka, Y., Shi, G.-Y., Kim, Y.-S., Ohashi, T., Tamura, K., and Zhang, D.: Balloon-borne observations of high aerosol concentrations near the summertime tropopause over the Tibetan Plateau, Atmos. Res., 84, 233-241, doi:10.1016/j.atmosres.2006.08.003, 2007.

Vernier, J.-P., Thomason, L. W., and Kar, J.: CALIPSO detection of an Asian tropopause aerosol layer, Geophys. Res. Lett., 38, L07804, doi:10.1029/2010GL046614, 2011a.

Vernier, J. P., Thomason, L. W., Pommereau, J. P., Bourassa, A., Pelon, J., Garnier, A., Hauchecorne, A., Blanot, L., Trepte, C., Degenstein, D., and Vargas, F.: Volcanic origin of the recent stratospheric aerosol trend, Geophys. Res. Lett., 38, L12807, doi:10.1029/2011GL047563, 2011 b.

Wang, P.-H., Minnis, P., McCormick, M. P., Kent, G. S., and Skeens, K. S.: A 6-year climatology of cloud occurrence frequency from SAGE II observations (1985-1990), J. Geophys. Res., 101, 29407-29429, 1996.

Wang, P.-H., Minnis, P., Wielicki, B. A., Wong, T., Cess, R. D., Zhang, M., Vann, L. B., and Kent, G. S.: Characteristics of the 1997/1998 El Niño cloud distributions from SAGE II observations, J. Geophys. Res., 108, 4009, doi:10.1029/2002JD002501, 2003. 